\title{
CLASSIFICAÇÃO CLIMATOLÓGICA DA ENERGIA POTENCIAL DISPONÍVEL PARA A CONVECÇÃO NA CIDADE DE BELÉM-PA
}

\author{
JOSIANE SARMENTO DOS SANTOS, MARIA AURORA SANTOS DA MOTA, EDSON JOSÉ \\ PAULINO DA ROCHA
}

\author{
Universidade Federal do Pará (UFPA), Instituto de Geociência, Belém, PA, Brasil. \\ josi_met@yahoo.com.br, aurora@ufpa.br, eprocha@fpa.br
}

Recebido Setembro de 2013 - Aceito Outubro de 2014

\begin{abstract}
RESUMO
A classificação climatológica da Energia Potencial Disponível para a Convecção (CAPE) foi estudada para verificar a possibilidade de ocorrer convecção e tipo de organização em Belém-PA, Brasil, associando-a com eventos extremos de precipitação (EEPRP). Para a análise da CAPE, utilizou-se dados de radiossondagens do DTCEA e de precipitação da estação do INMET, no período de 1987 a 2011. Observou-se que a atmosfera local apresenta condições favoráveis ao desenvolvimento de convecção profunda, pois a CAPE com valores maiores que $1000 \mathrm{~J} / \mathrm{kg}$ foi a mais frequente, representando $61 \%$ de todas radiossondagens. O valor de $1000 \mathrm{~J} / \mathrm{kg}$, segundo alguns autores, é o limite para convecção profunda. As classificações CAPE 2 e 3 apresentaram tendências significativas de aumento ao longo dos anos, enquanto a CAPE 1 diminuição. Essa situação provavelmente é resultado do aumento da temperatura do ar ocorrido na cidade, impactando o valor da CAPE. Os resultados também mostraram que as correlações entre a CAPE e EEPRP, a CAPE e os Índices Oceânico do Pacífico e Gradiente Inter-Hemisférico do Atlântico foram baixas. Então, a CAPE não é condição suficiente para formação de nuvens e precipitação, pois forçantes dinâmicas de meso e grande escala contribuem decisivamente para modular o clima da cidade.
\end{abstract}

Palavras-Chave: Amazônia; Convecção; Climatologia.

ABSTRACT: CLIMATOLOGICAL CLASSIFICATION OF AVAILABLE POTENTIAL ENERGY FOR CONVECTION IN THE CITY OF BELÉM-PA

The climatological classification of the Convection Available Potential Energy (CAPE) was studied in order to verify both the possibility of convection to start and the organizational type, in Belém (Pará - PA, Brazil), associating it to extreme precipitation events (EEPRP). For the CAPE analysis, DTCEA's radiosonde data and precipitation measurements from the INMET's stations, in the period from 1987 to 2011, we used. It was observed that the local atmosphere presented favorable conditions for the development of deep convection, because the most frequent CAPE values were higher than $1000 \mathrm{~J} / \mathrm{Kg}$, representing $61 \%$ of all radiosonde measurements. According to some authors, a CAPE value of $1000 \mathrm{~J} / \mathrm{Kg}$ is the deep convection limit. The CAPE 2 and 3 classifications presented significant increasing tendencies along the years, whereas CAPE 1 showed a decrease. This is probably due to the air temperature increased at the city, impacting the CAPE value. A low correlation between CAPE and EEPRP, as well as between CAPE and Pacific Ocean Index and the Atlantic Interhemispheric Gradient. was also shown. Therefore CAPE is not sufficient condition for the formation of clouds and precipitation, since meso and large scale dynamical forcing are relevant contributors to the climate modulation on the city.

Keywords: Amazon; Convection; Climatology. 


\section{INTRODUÇÃO}

O clima nos trópicos, em particular na Amazônia, é consequência de uma complexa combinação de vários sistemas meteorológicos, os quais interagem com a superfície e são responsáveis pela entrada local de umidade e energia na atmosfera, e dependente das propriedades dinâmicas e termodinâmicas da camada limite, que é a parte da troposfera diretamente influenciada pelos processos ou eventos que ocorrem na presença da superfície da Terra (Stull, 1988).

A atividade convectiva na região amazônica é um importante mecanismo de aquecimento da atmosfera tropical, pois a localização dessa região (entre $5^{\circ} \mathrm{N}$ e $10^{\circ} \mathrm{S}$ ) recebe no topo da atmosfera um valor máximo de $36,7 \mathrm{MJ} \cdot \mathrm{m}^{-}{ }^{2}$.dia- ${ }^{1}$, em dezembro/janeiro, e mínimo de 30,7 MJ.m- ${ }^{2}$.dia- ${ }^{1}$ em junho/ julho. Esses valores são reduzidos pela transmissão atmosférica, mas são, em média, de 15 MJ.m².- dia $^{-1}$ (Salati e Marques, 1984). Além da localização e disponibilidade de energia solar, o tipo de superfície, a cobertura de nuvens, o tipo de cobertura vegetal e albedo colaboram para o saldo de radiação (Charney, 1975). Essas características afetam a profundidade inteira da troposfera e servem para unir a camada limite com o resto da atmosfera. Portanto, contribuem para as definições das condições de tempo e clima na região.

Uma das maneiras de verificar a possibilidade de ocorrer convecção e o seu tipo de organização é fazer uma análise do ambiente termodinâmico, identificando a existência de instabilidade termodinâmica na atmosfera da região de estudo. A avaliação pode ser realizada por meio da análise de parâmetros objetivos, conhecidos como "índices de instabilidade", utilizados como ferramentas de auxílio à previsão do tempo, tanto em latitudes médias, como tropicais (Silva Dias, 2000; Nascimento, 2005; Lima, 2005).

Porém, não existem números exatos para a previsão de eventos de chuva. Mesmo os melhores parâmetros para previsão de tempo em escala tipicamente convectiva possuem alto índice de incerteza (Nascimento, 2005). Contudo, a previsão de tempo é indispensável para a sociedade, pois pode proteger e salvar vidas. Dessa maneira, estudar a eficiência dos índices de instabilidade, faz-se necessário.

Um dos mais consagrados índices para esta averiguação é a Energia Potencial Disponível para Convecção (CAPE), pois ela desempenha um papel importante no acionamento e na intensidade da convecção (Emanuel et al., 1994).

A CAPE é comumente empregada como: a) índice para previsão da possibilidade de ocorrer tempestades severas, pois fornece uma estimativa do limite superior para a velocidade vertical da corrente ascendente em uma nuvem convectiva (Doswell e Rasmussen, 1994); b) suas variações podem indicar mudanças climáticas globais (Ye et al., 1998; Gettelman et al., 2002); c) previsora de descargas elétricas atmosféricas/ intensidade de relâmpago na convecção profunda tropical (Williams et al., 1992, 2002); e d) estimativa de precipitação (McBride e Frank, 1999; Yano et al., 2005). Entretanto para todos itens mencionados ( $\mathrm{a}, \mathrm{b}, \mathrm{c}$ e d) existe um grau de incerteza tanto para o cálculo, como para a avaliação desejada, pois o cálculo da CAPE é altamente sensível à escolha da parcela de ar ascendente (Craven et al., 2002). Além disso, nem sempre a CAPE é fator determinante para o desenvolvimento da convecção úmida profunda e ocorrência de precipitação (PRP), pois muitas vezes existem valores altos da CAPE e nenhuma PRP é observada (Mota e Nobre, 2006).

Tavares e Mota (2012), analisaram condições termodinâmicas de eventos extremos de PRP em Belém-PA, durante a estação chuvosa, para o período de 1987-2006, utilizando dados de radiossondagens do Destacamento de Controle do Espaço Aéreo de Belém (DTCEA-BE) no Aeroporto Internacional Val de Cans na cidade de Belém-PA, e PRP da estação da EMBRAPA. Na avaliação dos índices de instabilidade da atmosfera, constatou-se que a convecção severa pode se desenvolver mesmo com baixos valores de CAPE (menor que $1000 \mathrm{~J} / \mathrm{kg}$ ), pois a PRP extrema, do período estudado, também esteve associada aos sistemas de meso e grande escala (fatores dinâmicos) que, possivelmente se sobrepuseram à escala local. Os autores concluíram ainda que as condições termodinâmicas são necessárias, mas não são as únicas responsáveis pela formação da convecção úmida profunda com precipitações extremas na estação chuvosa da cidade. Então, os fatores dinâmicos têm papel importante para formação e desenvolvimento das nuvens precipitantes na região, pois ajudam as parcelas a alcançar o nível de condensação por levantamento (NCL), que é a base das nuvens atuando como "gatilho" para o disparo da convecção úmida profunda (Lima, 2005; Mota e Nobre, 2006).

A presente pesquisa foi realizada na cidade de Belém-PA, que está situada no leste da Amazônia e possui duas estações bem definidas: chuvosa de dezembro a maio, e menos chuvosa de junho a novembro (Figueroa e Nobre, 1990; Marengo et al., 2001; e De Souza e Ambrizzi, 2002). A definição das estações, chuvosa e menos chuvosa, é consequência, principalmente, da migração latitudinal da Zona de Convergência Intertropical (ZCIT), que durante o verão austral está posicionada mais abaixo da linha do Equador, podendo alcançar até $5^{\circ} \mathrm{S}$ de latitude, provocando intensas chuvas nessa região, enquanto no inverno austral está mais ao norte, podendo alcançar até $10^{\circ} \mathrm{N}$, e como consequência ocorre redução das chuvas na Amazônia (Citeau et al., 1985, 1988a,b; Uvo e Nobre, 1989; Waliser e Gautier, 1993; Cavalcanti et al., 2009). Além da ZCIT, a região também é influenciada por outros sistemas meteorológicos de meso escala intensificadores de chuva, como as Linhas de Instabilidade (LI), 
que são responsáveis por cerca de $45 \%$ da chuva no período menos chuvoso (Cohen, et al., 1989 e Cavalcanti et al., 2009).

A variabilidade da PRP também pode ser alterada pelo ciclo do El Niño-Oscilação Sul (ENOS), sobre o Pacífico e as fases do Gradiente Meridional e Inter-hemisférico das anomalias de Temperatura da Superfície do Mar (TSM) na bacia intertropical do Atlântico, conforme estudos feitos por diversos autores, dentre eles (Nobre e Shukla, 1996; De Souza et al., 2000; Pezzi e Cavalcanti, 2001; Liebmann e Marengo, 2002). De qualquer maneira, um sistema de tempo produtor de PRP intensa pode se tornar evento extremo e estar relacionado à atividade convectiva, o qual é resultado da interação de mecanismos físicos de diferentes escalas (Rockwood e Maddox, 1988).

Os eventos climáticos extremos causam prejuízos e transtornos para sociedade em qualquer região do mundo, e na região Amazônica não é diferente das demais. Um exemplo de evento extremo ocorrido na Amazônia foi a seca de 2005, que esteve relacionada ao aquecimento anormal das águas do Atlântico Tropical Norte, e provocou grandes incêndios, principalmente no sudoeste da Amazônia (Marengo et al., 2008a,b). Como consequência dos incêndios, o tráfego aéreo foi afetado com o fechamento do aeroporto internacional de Rio Branco no estado do Acre. Escolas, empresas foram fechadas devido à fumaça, e muitas pessoas tiveram que ser atendidas nos hospitais devido à inalação de fumaça (Marengo et al., 2008 a,b; Zeng et al., 2008, Cox et al., 2008).

Então, a presente pesquisa foi desenvolvida considerando a importância que a atividade convectiva exerce sobre o clima da cidade. Com o objetivo de estabelecer a classificação climatológica da CAPE (25 anos), tendência da CAPE, relacionar a CAPE com os Índices Oceânico do Pacífico e Gradiente Inter-Hemisfério no Atlântico, fazer correlação entre CAPE e eventos extremos de precipitação, verificando dessa maneira, a importância desse índice para previsão de eventos extremos de PRP.

\section{DADOS E METODOLOGIA}

\subsection{Dados}

No presente trabalho foram utilizados dados de radiossondagens do período de 1987 a 2011 (25 anos), com total de 9.233 radiossondagens obtidos do sítio do Departamento de Ciências Atmosféricas da Universidade de Wyoming (http:// weather.uwyo.edu/upperair/sounding.html). Os horários de lançamento são às 00 e 1200 UTC, porém a sondagem das 0000 UTC só começou a ser lançada a partir do ano 1996, na estação meteorológica de ar superior do Destacamento de Controle do Espaço Aéreo de Belém (DTCEA-BE) no Aeroporto Internacional Val de Cans na cidade de Belém-PA ( $1^{\circ} 23^{\prime} \mathrm{S}$ e $\left.48^{\circ} 29^{\prime} \mathrm{W}\right)$.
Os dados de precipitação (PRP) são da estação de Belém (código da estação meteorológica: 82191, latitude de $01^{\circ} 43^{\prime} \mathrm{S}$ e longitude de $48^{\circ} 43^{\prime} \mathrm{W}$ ) do Instituto Nacional de Meteorologia (INMET), coletados todos os dias às 1200 UTC, ou seja, acumulada durante 24 horas.

Os dados do Índice Oceânico Niño (ION) no Pacífico, na região $3.4\left(05^{\circ} \mathrm{N}-05^{\circ} \mathrm{S}, 120^{\circ}-170^{\circ} \mathrm{W}\right)$ e Gradiente Interhemisfério no Atlântico (GIHA) estão disponíveis no portal da National Oceanic and Atmospheric Administration (http:// www.cpc.ncep.noaa.gov).

\subsection{Metodologia}

Testes estatísticos como média aritmética simples, tendência da série, periodicidade, distribuição de frequência, técnica dos decis e correlação foram utilizados para o desenvolvimento do estudo.

As médias aritméticas foram usadas para averiguar a variação da PRP do período estudado, ou seja, conhecer o comportamento das chuvas dessa série e fazer a média anual do ION e GIHA, identificando as anomalias positivas e negativas desses índices, que estão descritas no portal da National Oceanic and Atmospheric Administration (http://www.cpc.ncep.noaa.gov).

A tendência da CAPE na série analisada foi notada através do teste de Mann-Kendall (Sneyers, 1992), o qual mostra se a sucessão de valores $\mathrm{X} 1, \mathrm{X} 2, \ldots, \mathrm{Xn}$ de uma série temporal ocorre de forma independente e identicamente distribuída da seguinte maneira:

$\mathrm{H}_{0}=$ Não há tendência na série

$\mathrm{H}_{1}=$ Há tendência na série

Sendo que $\mathrm{H}_{0}$ é a hipótese nula, ou seja, os valores da série são dependentes. A hipótese $\mathrm{H}_{0}$ é rejeitada quando o valor de tendência encontrado tem nível de significância maior que 0,05 . A hipótese alternativa $\mathrm{H}_{1}$ informa que os dados são independentes e identicamente distribuídos.

Para a verificação a existência de periodicidade(s) significativa(s) na série de dados da CAPE, foram realizados os testes de Fisher estendidos (Shimshoni, 1971), ou seja, foi verificado se existiu alguma periodicidade na série de dados da CAPE.

A avaliação e classificação das sondagens (mensal e anual), utilizando CAPE, foram realizadas segundo a proposta de Rogash e Racy (2002), verificando a frequência de ocorrência das classificações, conforme apresentado na Tabela 1, que relaciona a atividade convectiva com os valores da CAPE. Esses autores definiram os valores de CAPE que podem conduzir a eventos extremos, e fizeram uma estatística das condições termodinâmicas de ambientes precursores de tornado e enchentes repentinas nos Estados Unidos num período de 40 anos (1955-1995), e obtiveram que a menor CAPE encontrada 
foi de $1.400 \mathrm{~J} / \mathrm{kg}$ e a maior, de $3.200 \mathrm{~J} / \mathrm{kg}$. Esses valores de referência da CAPE foram propostos nas latitudes médias e serão utilizados neste trabalho como referência em latitudes tropicais para avaliar a relação entre EEPRP e a CAPE.

A CAPE foi calculada através da Equação 1, proposta por Emanuel (1994):

$$
C A P E=\int_{N C E}^{N E} g\left(\frac{T_{v p}-T_{v a}}{T_{v a}}\right) d z
$$

onde, NCE é o Nível de Convecção Espontânea; NE o Nível de Equilíbrio; $T_{p}$ é a Temperatura virtual da parcela, $T_{v a}$ é a Temperatura virtual do ambiente, que se encontra entre a base da nuvem (NCE) e o topo da nuvem (NE); g é a aceleração da gravidade $\left(9,8 \mathrm{~m} \cdot \mathrm{s}^{2}\right)$ e dz é a espessura da camada (m).

Devido ao fato de que a CAPE é sensível à escolha da parcela, foi considerada a temperatura do virtual do primeiro nível acima do nível da superfície. Os valores de temperatura virtual da atmosfera próximos à superfície podem conter variações provocadas pela altura da sonda em relação ao solo, no momento do lançamento, e eventuais modificações nas características da superfície local, como grama e asfalto. Sendo assim, o nível considerado está a aproximadamente 10 milibares acima do nível médio do mar.

A CAPE é a medida da integral na vertical da força de flutuabilidade da parcela entre os níveis de convecção espontânea (NCE) e equilíbrio (NE). Fisicamente, a CAPE é a medida da energia cinética máxima por unidade de massa de ar, proporcionada pela ascensão adiabática (ou pseudoadiabática) de ar úmido da camada limite, o que pode fornecer o limite superior para a velocidade vertical da corrente ascendente em uma nuvem convectiva (Ye et al., 1998).

O monitoramento da PRP (em casos de estiagem ou de PRP elevada) pode determinar se o evento é extremo. Isso porque a PRP é um dos principais elementos meteorológicos para análise do clima de uma região, pois constitui a fase aérea do ciclo hidrológico e influencia no comportamento de outras variáveis como temperatura, vento e umidade (Marengo e Hastenrath, 1993).

Para a análise de EEPRP foi utilizada a técnica dos decis, que pertence à metodologia das separatrizes (quartil, decil e percentil), descritas por Xavier et al. (2007). Nesse método, um conjunto de dados (ex, precipitação diária) é ordenado de forma crescente e o valor médio que divide a série em duas partes iguais é a mediana. Por extensão desse conceito, pode-se pensar nos valores que dividem o conjunto em dez partes iguais, que serão denominadas de decis e são representados por D1, D2, ...D9. O quinto decil corresponde à mediana, e o nono decil fornece $10 \%$ dos valores mais extremos da série. Os decis foram calculados pela equação de Xavier et al. (2007):

$$
D k=l i+\left[\frac{\frac{k \sum f i}{10}-f a n t}{f i}\right] h
$$

onde, $\mathrm{D} k$ é o decil que se pretende calcular, $\mathrm{h}$ a amplitude da distribuição, $l i$ o limite inferior da classe, fi a frequência da classe da posição do decil, fant a frequência da classe anterior do decil e $\mathrm{k}$ o número de ordem do decil.

Após a aplicação da técnica foi considerado EEPRP, chuvas maiores que 50 milímetros $(\mathrm{mm})$ por dia, se encontrando no nono decil, o qual apresenta os casos mais raros de PRP extrema, aqueles que, embora sejam em número muito reduzido, correspondem a $10 \%$ do total de eventos de chuva de toda a série.

As técnicas de seleção de eventos extremos foram utilizadas por diversos autores como, por exemplo, Xavier e Xavier (1999), que realizaram um estudo sobre as chuvas dos meses de fevereiro a maio de 1964-1998 em diferentes áreas no estado do Ceará, e encontraram na região do sertão evento extremo de seca de $361 \mathrm{~mm}$ e no litoral evento extremo de precipitação de $1179,5 \mathrm{~mm}$, ambos observados no quadrimestre do período chuvoso da região. Tavares e Mota (2012) utilizaram a técnica dos decis proposta por Xavier et al. (2007) para análise

Tabela 1 - Valores de CAPE

\begin{tabular}{|c|c|c|}
\hline CAPE & Condições Associadas & Neste Trabalho \\
\hline$>0-1000$ & $\begin{array}{c}\text { Limite para formação da } \\
\text { convecção profunda }\end{array}$ & CAPE1 \\
\hline $1000-2500$ & $\begin{array}{c}\text { Convecção profunda } \\
\text { moderada }\end{array}$ & CAPE2 \\
\hline $2500-4000$ & Convecção profunda forte & CAPE3 \\
\hline$>4000$ & Convecção Severa & CAPE4 \\
\hline
\end{tabular}

Fonte: Rogash e Racy (2002) 
de evento extremo de precipitação na cidade de Belém-Pa, durante 21 anos (1987-2007) para o período chuvoso, onde o nono decil refere-se às chuvas maiores do que $67,1 \mathrm{~mm} / \mathrm{d}$. Essa diferença de valores de EEPRP encontrada entre as pesquisas está relacionada com o tamanho das amostras, além de que, os autores só utilizaram os dados de precipitação do período chuvoso da cidade.

Para conhecer possíveis correlações entre as frequências da CAPE e EEPRP, e as classificações da CAPE com o ION e GIHA, foi realizado teste de correlação, medindo a associação entre os parâmetros que foram quantificados através do coeficiente de correlação linear de Pearson $(r)$. O coeficiente $r$ varia de -1 a 1 . O sinal indica direção positiva ou negativa do relacionamento e o valor sugere o grau de relacionamento entre as variáveis.

\section{RESULTADOS E DISCUSSÃO}

A variação da PRP mensal no período estudado para Belém-PA é apresentada na Figura 1, em que a média mensal do período foi de $259 \mathrm{~mm}$ (sendo representada por uma linha reta horizontal sobre o gráfico). Pode ser observado que a cidade tem duas estações bem definidas: a) chuvosa (dezembro a maio), e b) menos chuvosa (junho a novembro), sendo que a chuvosa corresponde a mais de $70 \%$ do total anual, e a menos chuvosa corresponde a menos de 30\% do total anual (Costa, 1998; Silva Junior et al., 2012). O período chuvoso é caracterizado pela influência da forçante dinâmica de grande escala, ZCIT, que em sua posição mais ao sul do equador nesses meses (Citeau et al.,
1985, 1988a,b; Uvo e Nobre,1989; Waliser e Gautier, 1993), provoca ocorrência de grande quantidade de precipitação na região amazônica (Marengo et al., 2001; De Souza e Ambrizzi, 2002). No período menos chuvoso, a ZCIT está mais ao norte, então há uma redução das chuvas, porém, as LI que atuam na região contribuem com $45 \%$ das chuvas observadas (Cohen et al., 1989).

Foi realizada uma análise para tendência do valor da CAPE ao longo dos anos estudados, utilizando o teste de MannKendall. A análise da série apresentou tendência de aumento nos valores da CAPE, com nível de significância de 0,174 , superior ao limite de 0,05 para rejeitar a tendência de uma série (Sneyers, 1992). Estudo semelhante realizado por Murugavel et al., (2012) para região da Índia, utilizando dados de 25 anos (1984-2008), também encontrou tendência de aumento do valor da CAPE $(0,8 \sim 0,9)$ na estação pré Monção de algumas regiões da Índia, constatando que os maiores valores da CAPE estavam associados ao aumento no teor de umidade em baixos níveis e diminuição da temperatura em altos níveis nos últimos 25 anos. Diferentemente da região estudada por Murugavel et al. (2012), Belém-PA não apresentou a tendência da CAPE expressiva. As variáveis temperatura e vapor d'água, que impactam no valor da CAPE, não têm variabilidade temporal grande, apesar da grande quantidade de vapor d'água nos baixos níveis da troposfera (Superfície-925 hPa), e gradiente vertical de temperatura muito forte (Ananias et al., 2010).

Desta maneira, fez-se necessário uma análise mais detalhada da tendência dos valores da CAPE para a cidade. Então foram avaliadas: a) periodicidade da (APE b) a distribuição

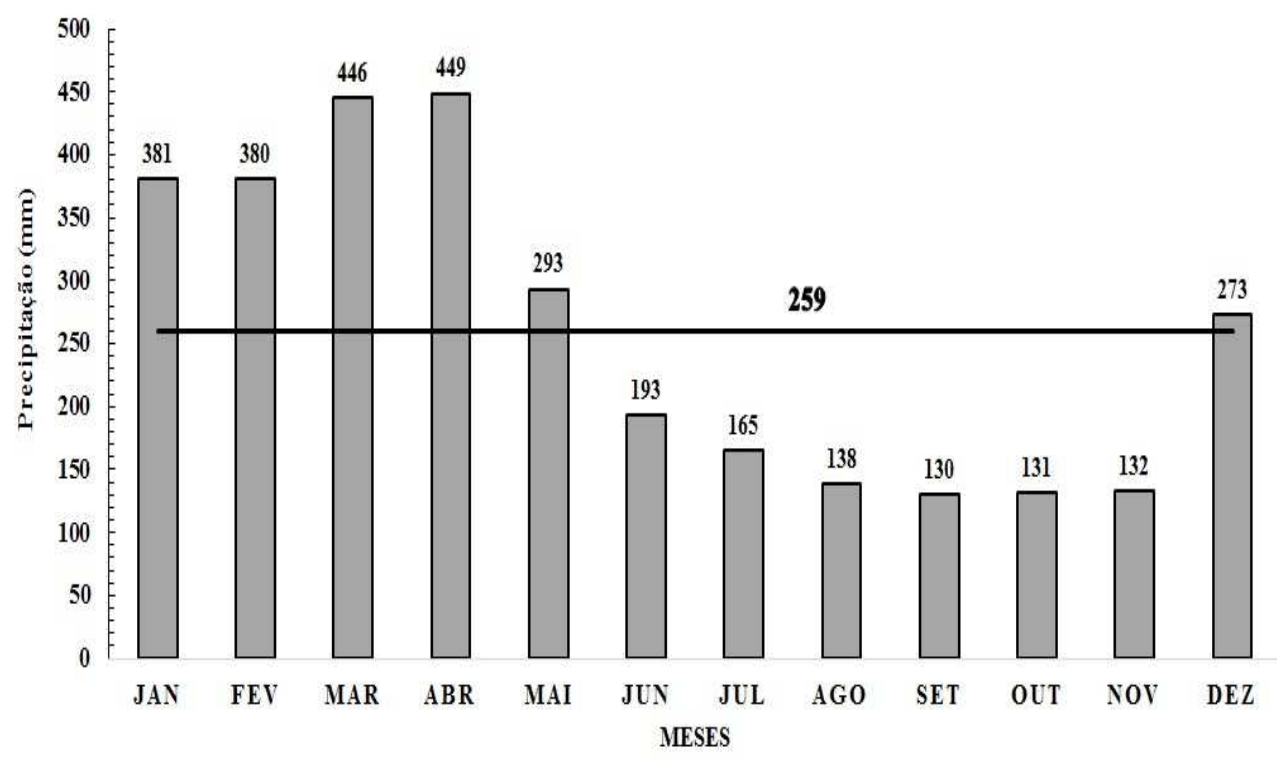

$\square$ Media Mensal PRP 25 anos -Media total 25 anos

Figura 1 - Variação da precipitação média mensal, no período de 1987 a 2011 para Belém-PA. 


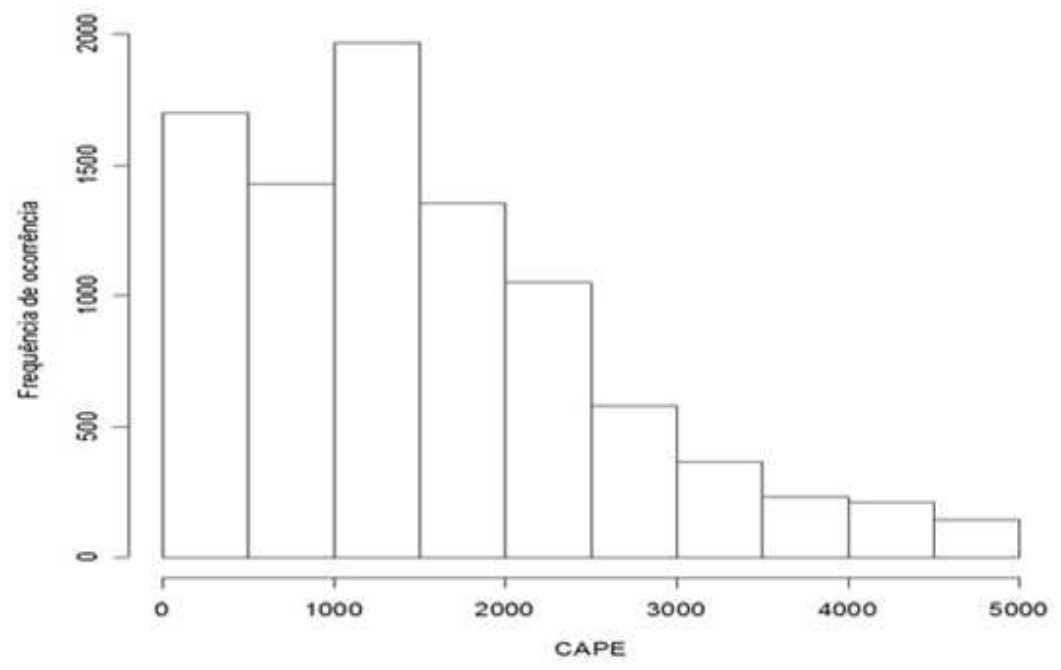

Figura 2 - Frequência de ocorrência da CAPE no período estudado (1987-2011) para Belém-PA.

de frequência da série total dos valores da CAPE (Figura 2); c) classificação da CAPE (Figura 3), conforme sugerido por Rogash e Racy (2002), e d) distribuição de frequência anuais e mensais das classificações da CAPE (Figuras 4 e 5).

Quando realizado o teste de Fisher estendido, a CAPE apresentou periodicidade de 4 dias, porém para a CAPE não é significativa. Isto porque, a CAPE é a energia disponível, então se a convecção úmida profunda se formar, a nuvem precipitante consome a CAPE, na medida em que ela necessita dos movimentos verticais ascendentes para o desenvolvimento da nuvem. Com isso, a convecção profunda durante a precipitação, cria correntes descendentes que estabilizam o ambiente localmente, implicando em menor valor de CAPE, podendo chegar a zero.

Na distribuição de frequência da série total dos valores da CAPE (Figura 2), a maior frequência para os 25 anos estudados, foram os valores da CAPE ocorrendo entre 1000 a $1500 \mathrm{~J} / \mathrm{kg}$, que estão contidos no intervalo da categoria CAPE 2 (Tabela 1), a chamada CAPE $2\left(1000\right.$ a $\left.2500 \mathrm{Jkg}^{-1}\right)$ teve maior frequência (44\% das ocorrências), sendo que $61 \%$ das sondagens tiveram valores de CAPE acima de $1000 \mathrm{~J} / \mathrm{kg}$, como pode ser observado na Figura 3, que destaca as 4 classificações sugeridas por Rogash e Racy (2002), sendo assim, a análise evidencia que a CAPE de Belém tem na maior parte do tempo valores entre 1000 e $2500 \mathrm{~J} / \mathrm{kg}$.

Williams e Rennó (1993) quando avaliaram os dados do experimento Atmospheric Boundary Layer Experiments (ABLE-2b), ocorrido nos meses de março a maio de 1987, para Belém-PA, utilizando três metodologias diferentes para o cálculo do valor da CAPE, também encontraram maior frequência para os valores da CAPE no intervalor 1000-2500 $\mathrm{J} / \mathrm{kg}$. Então, a atmosfera de Belém-PA apresenta condições favoráveis para suportar a convecção profunda, principalmente a convecção úmida profunda moderada (CAPE 2), pois a CAPE presente numa atmosfera convectiva em condições de equilíbrio estável é uma medida da quantidade de dissipação mecânica de energia contida nessa atmosfera, e um valor expressivo da CAPE, (maior que $1000 \mathrm{~J} / \mathrm{kg}$ ), é necessário para sustentar as circulações convectivas, devido às perdas dissipativas, segundo alguns autores, dentre eles Rennó e Ingersoll (1996).

A Figura 4 mostra a frequência mensal dos valores da CAPE. A frequência mensal das categorias CAPE 2, 3 e 4 foi maior no período menos chuvoso (junho a novembro). Esse fato também foi evidenciado nos estudos de Machado et al. (2002) e decorre do aumento na temperatura do ar com diminuição da PRP. Então, a maioria dos eventos de PRP fica mais dependente

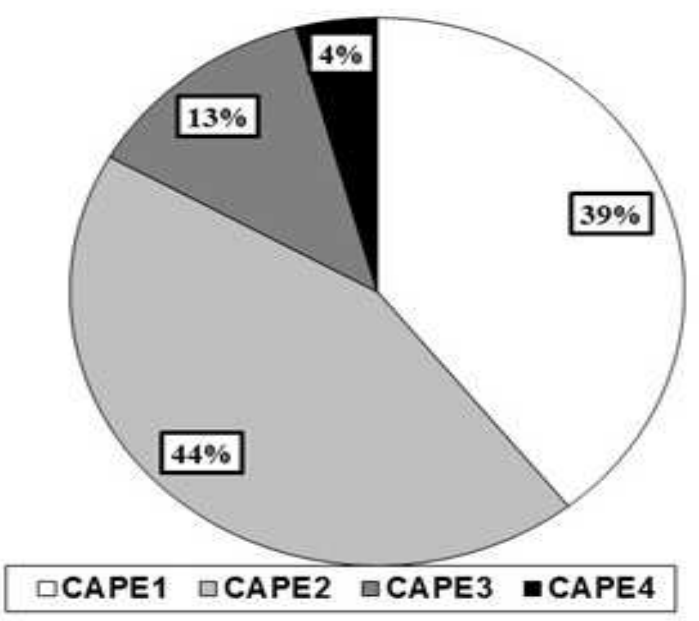

Figura 3 - Classificação das sondagens conforme a definição da CAPE, em Belém - PA (1987-2011). 
da forçante termodinâmica, pois a forçante dinâmica que atua neste período é de meso-escala, brisas e LI. Portanto, na estação menos chuvosa, os valores da CAPE chegam a ter no máximo entre 4000 e $5000 \mathrm{~J} / \mathrm{kg}$, o que contribui para produzir nuvens de grande desenvolvimento vertical, que são altamente eletrificadas, com ventos fortes e chuvas com duração de até 2 horas (Rutledge et al., 2000).

Ainda na análise da Figura 4, pode-se perceber na estação chuvosa que a categoria CAPE 4 apresenta menor frequência em relação a estação menos chuvosa, isso acontece porque a forçante dinâmica de grande escala (ZCIT) atua intensamente, o que acelera as parcelas mais rapidamente, implicando em atingir o nível de equilíbrio (NE) mais cedo, ou seja, o NE é mais baixo, consequentemente valor de CAPE é menor. As nuvens têm menor desenvolvimento vertical, porém, as chuvas são mais abundantes com duração maior que 2 horas (Hastenrath e Lamb, 1997). Além disso, quando existe forçante dinâmica, ocorrem eventos de chuvas no período noturno (noite e madrugada). Sendo assim, a estação chuvosa na Amazônia apresenta características de regimes tropicais marítimos, isto é, menor valor da CAPE, correntes ascendentes mais fracas e reduzida atividade elétrica (Williams et al., 2002; Petersen et al., 2002; Petersen et al., 2006).

Para verificar a tendência da CAPE (Figura 5), obter a correlação entre CAPE e EEPRP, e determinar a relação entre CAPE e ION e GIHA (Figura 7), utilizou-se a série dos dados das sondagens somente para as 1200UTC, pois nos anos de 1987 a 1995 só foram realizadas radiossondagens no horário das 1200 UTC.

$\mathrm{Na}$ Figura 5, pode ser observado que existe um aumento da frequência dos valores das categorias CAPE 2 e 3 no período estudado, e diminuição dos valores da CAPE 1, principalmente a partir do ano 2000. Realizado então o teste de Mann-Kendall, o nível de significância de aumento para as CAPE 2, 3 foi respectivamente 0,27 e 0,32, enquanto a CAPE 1 mostrou tendência de diminuição de 0,18 . Então, como estas tendências são maiores que 0,05 , a hipótese nula é rejeitada. Considerando que os valores da CAPE são dependentes da umidade e temperatura na camada limite, uma provável causa para a maior frequência da CAPE 2, em Belém-PA, seria o aumento de $0,8^{\circ} \mathrm{C}$ na temperatura do ar na cidade evidenciada nos estudos de Xavier et al. (2002), pois a camada limite sofre alterações devido às variações nos fluxos de calor e umidade que ocorrem nela (Donner e Phillips, 2003; Zhang, 2003a,b).

A Figura 6 mostra a frequência anual dos EEPRP no período estudado, e a média foi de 7 EEPRP por ano, com o máximo de 16 (2011) e o mínimo de 2 (2003). A tendência (linha preta) dos EEPRP evidencia um aumento da frequência dos EEPRP a partir do ano 2000, realizado então o teste de MannKendall, o nível de significância foi de 0,18 , que é maior do que 0,05 , ou seja, a hipótese nula é rejeitada. É importante ressaltar que a maioria dos eventos extremos ocorreu no período chuvoso (Tabela 2), que tem influência principalmente do sistema meteorológico de grande escala, ZCIT (Figueroa e Nobre, 1990; Marengo et al., 2001; e De Souza e Ambrizzi, 2002).

Todavia, embora a CAPE 2 e 3 também tenham apresentado tendência de aumento (Figura 5), a relação entre as frequências da CAPE (classificadas) e EEPRP (PRP > 50 $\mathrm{mm}$ ) apresentou correlação apenas para a CAPE 3, de 0,35, que apesar de positiva, é fraca (Cohen, 1988). Então, os valores altos da CAPE não estão diretamente relacionados com EEPRP, pois CAPE é condição necessária para formação da nuvem, porém não suficiente para o desenvolvimento da convecção profunda

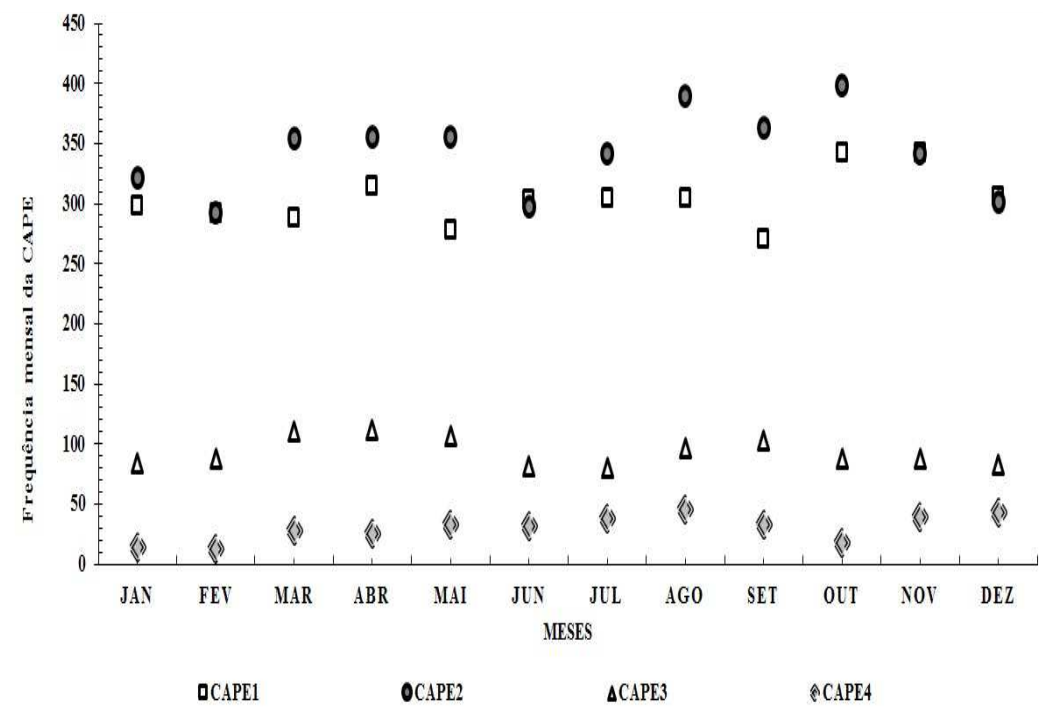

Figura 4 - Frequência mensal das 4 classificações da CAPE, para Belém-PA no período de 1987 a 2011. 


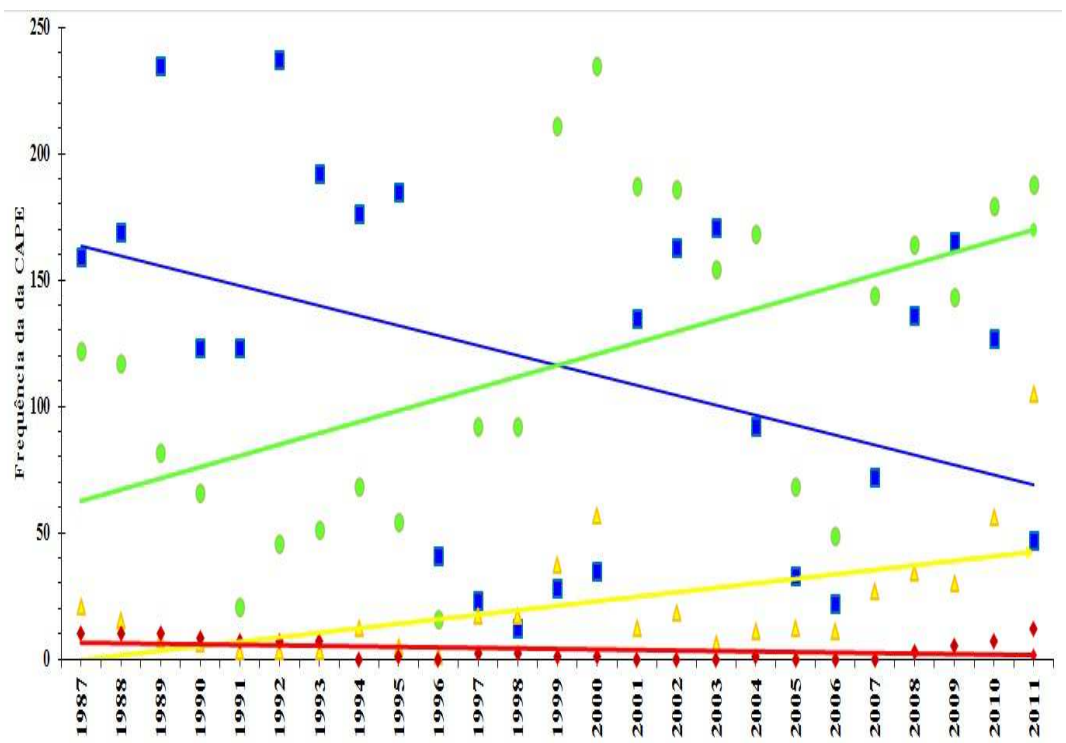

Figura 5 - Frequência anual e tendência das 4 categorias da CAPE para a série analisada em Belém-PA nos anos de 1987 a 2011.

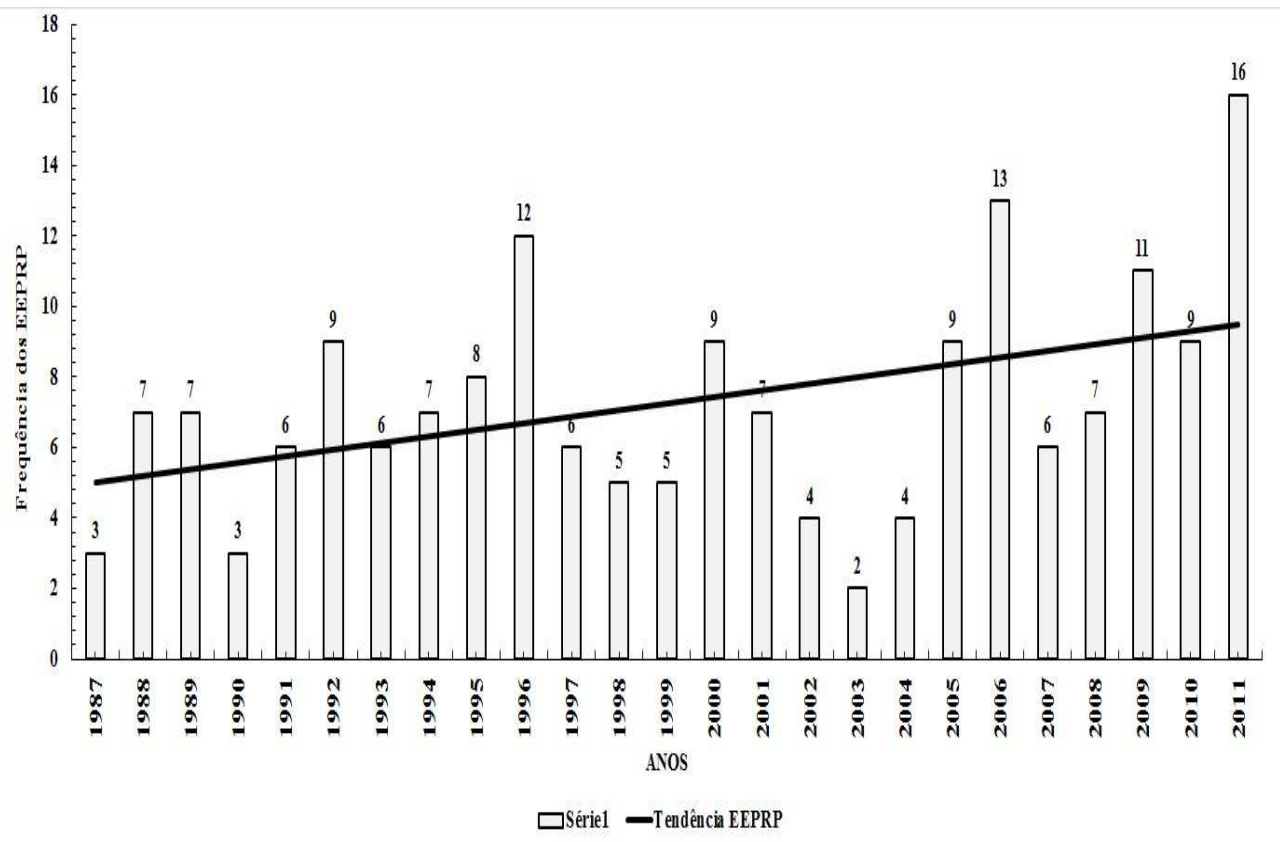

Figura 6 - Frequência anual e tendência dos EEPRP, para Belém-PA no período de 1987 a 2011.

(Mota e Nobre, 2006), requerendo a forçante dinâmica para o disparo da convecção ("gatilho"), ou seja, algum mecanismo que produza levantamento deste ar instável, para que a convecção seja iniciada (Mota, 2004; Lima 2005). Dessa maneira, no período chuvoso (dezembro a maio) a CAPE $4(>4000 \mathrm{~J} /$ $\mathrm{kg}$ ) é menos frequente que no período menos chuvoso, com quantidade de EEPRP de 149 e 32 eventos, respectivamente (Tabela 2). As diferenças de CAPE e EEPRP entre os dois períodos é devido aos sistemas de meso e grande escala atuantes na região, que agem, ora acelerando os sistemas locais, provocando fortes chuvas, ora enfraquecendo-os, diminuindo a quantidade de precipitação.

A Figura 7 mostra a frequência das categorias da CAPE e a variação média do ION e GIHA no período de 1987 a 2011. Nota-se que os anos de 1987 e 1997 foram períodos de ocorrência de El Niño (GGWS, 2014) com ION, em média, mais positivo nesses anos, ou seja, apresentou anomalia positiva, situação semelhante pode ser observada nos anos de 1992 e 1994, e nos anos de 2000 e 2003, onde o ION apresentou anomalia positiva e a CAPE 1 apresenta uma frequência maior. 
Tabela 2 - Frequência mensal dos EEPRP, para Belém-PA no período de 1987 a 2011.

\begin{tabular}{|c|c|c|c|c|c|c|c|c|c|c|c|c|c|}
\hline ANOS & Jan & Fev & Mar & Abr & Mai & Jun & Jul & Ago & Set & Out & Nov & Dez & SOMA \\
\hline 1987 & 1 & 1 & 1 & & & & & & & & & & 3 \\
\hline 1988 & 1 & & 2 & 2 & & 1 & & 1 & & & & & 7 \\
\hline 1989 & & 1 & & 2 & 2 & & & & 1 & & & 1 & 7 \\
\hline 1990 & & 1 & & 1 & & & & 1 & & & & & 3 \\
\hline 1991 & 2 & 2 & 1 & & 1 & & & & & & & & 6 \\
\hline 1992 & 2 & 6 & & 1 & & & & & & & & & 9 \\
\hline 1993 & 4 & 1 & 1 & & & & & & & & & & 6 \\
\hline 1994 & 1 & 1 & 1 & 1 & 1 & 1 & & & & & & 1 & 7 \\
\hline 1995 & 1 & 1 & & & 3 & & & & & 1 & 1 & 1 & 8 \\
\hline 1996 & & 2 & 3 & 3 & 1 & 2 & & & & & 1 & & 12 \\
\hline 1997 & 1 & 2 & 3 & & & & & & & & & & 6 \\
\hline 1998 & & 1 & & 2 & & & & & & & 1 & 1 & 5 \\
\hline 1999 & & & 1 & 2 & 1 & & & & & & & 1 & 5 \\
\hline 2000 & 1 & 2 & & 2 & 2 & & & & & & & 2 & 9 \\
\hline 2001 & & & 1 & 1 & & & 3 & & & & 1 & 1 & 7 \\
\hline 2002 & 1 & & 1 & 1 & & 1 & & & & & & & 4 \\
\hline 2003 & & & & 1 & & & & & & & & 1 & 2 \\
\hline 2004 & 2 & & 2 & & & & & & & & & & 4 \\
\hline 2005 & & & 3 & 1 & 1 & & 1 & & & 1 & & 2 & 9 \\
\hline 2006 & 1 & & 4 & & 3 & & & 2 & 1 & & & 2 & 13 \\
\hline 2007 & 1 & 1 & 1 & 1 & & & & & & & & 2 & 6 \\
\hline 2008 & & 1 & 2 & 1 & & 1 & & 2 & & & & & 7 \\
\hline 2009 & 1 & & 2 & 3 & 1 & 2 & & & & 1 & & 1 & 11 \\
\hline 2010 & 3 & 2 & & & 1 & 2 & & 1 & & & & & 9 \\
\hline 2011 & 3 & 2 & 1 & 5 & 3 & & & 1 & & & 1 & & 16 \\
\hline SOMA & 26 & 27 & 30 & 30 & 20 & 10 & 4 & 8 & 2 & 3 & 5 & 16 & 181 \\
\hline MÉDIA & 2 & 2 & 2 & 2 & 2 & 1 & 2 & 1 & 1 & 1 & 1 & 1 & 7 \\
\hline & & & & & & & & & & & \\
\hline
\end{tabular}

Em 1988 e 1999 foram períodos de La Niña, com o ION em média negativo (anomalia negativa). O GIHA esteve, em média, mais positivo (anomalia positiva) nos anos 2005 e 2007 e mais negativo (anomalia negativa) em 1989, 1990 e 1994.

Nos anos de El Niño, a CAPE $1(<1000 \mathrm{~J} / \mathrm{kg})$ foi mais frequente em 1987 e CAPE $2(1000$ a $2500 \mathrm{~J} / \mathrm{kg})$ em 1997 , indicando que o El Niño influenciou nos valores da CAPE para Belém. Uma justificativa para este fato é que o valor da CAPE é calculado pela diferença entre a temperatura da parcela e a temperatura do perfil vertical da atmosfera (Tvp-Tva), portanto, se o perfil vertical do ambiente estiver mais quente, a diferença será menor, implicando no menor valor de CAPE. Isso porque, o fenômeno El Niño aquece e seca o ambiente na região, através das correntes descendentes, provocando menor valor de CAPE. Assim, a atmosfera tem menos energia disponível para a formação da convecção úmida profunda, necessitando de uma forçante dinâmica ("gatilho") forte para o desenvolvimento de nuvens precipitantes. Um exemplo é o perfil vertical da temperatura, em 1987, que esteve mais quente que a média dos 25 anos estudados (Figura 8). Os impactos do El Niño sobre a precipitação da região amazônica são amplamente investigados. Esses estudos demonstram que as condições da TSM do Oceano Pacífico, quando estão maiores que a média, influenciam significativamente na circulação troposférica zonal da célula de Walker (Kousky et al., 1984; Kayano e Moura, 1986; Marengo, 1992; Pezzi e Cavalcanti, 2001; De Souza e Ambrizzi, 2002), inibindo a atividade convectiva da ZCIT e consequentemente, causam déficit expressivo de PRP na região (Coelho et al., 1999; De Souza et al., 2000), em particular o ano de 1987 (Sousa, 2003).

Os anos 1988 e 1999 tiveram influência do fenômeno La Niña, (GGWS, 2014) a categoria CAPE 1 foi mais frequente para 1988 e CAPE 2 para 1999. O fenômeno La Niña através das correntes ascendentes esfria e umedece $\mathrm{o}$ ambiente na região de estudo, além de acelerar o deslocamento da parcela, devido aos ventos alísios de nordeste ficarem mais intensos e a ZCIT ter posição mais ao sul do Equador. Sendo assim, a forçante dinâmica é muito forte. Em 1999 o perfil vertical de temperatura está mais frio que a média dos 25 anos estudados (Figura 8). Desse modo, o transporte de umidade para a Amazônia é mais ativo, o que aumenta a formação das nuvens convectivas na região, porém essas nuvens apresentam menores topos, devido 
às parcelas atingirem mais rapidamente ao NE. Todavia, a quantidade e duração da precipitação é maior (Kousky et al., 1984; Kayano e Moura 1986; Marengo, 1992; Pezzi e Cavalcanti, 2001; De Souza e Ambrizzi, 2002), o que provoca diminuição na CAPE.

O GIHA esteve, em média, mais positivo (anomalia positiva) nos anos 2005 e 2007 com a CAPE 2 (1000 a 2500 $\mathrm{J} / \mathrm{kg}$ ) mais frequente (Figura 7). Contudo, não apresenta qualquer relação com aos valores da CAPE, visto que CAPE 2 é uma característica frequente na cidade (Figura 3). Isso significa dizer que as condições do Oceano Atlântico Sul (OAS), mais quente, não interferem na CAPE de Belém, pois quando o GIHA tem valor positivo, significa que o Oceano Atlântico Norte está mais quente que o OAS (Nobre e Shukla, 1996). O GIHA com anomalia positiva no ano de 2005 causou evento extremo de seca no sudoeste da Amazônia, como citado anteriormente.

\section{CONCLUSÃO}

Neste estudo evidenciou-se que a atmosfera de Belém-PA tem altos valores de CAPE, pois $44 \%$ das classificações da CAPE estiveram entre 1000 e $2500 \mathrm{~J} / \mathrm{kg}$, caracterizando potencial para forte atividade convectiva na região. Então, se existir a forçante dinâmica (gatilho) a convecção úmida profunda se desenvolve, podendo assim, ocorrer precipitação.
A tendência de aumento nos valores da CAPE foi muito pequena com periodicidade não significativa. $\mathrm{E}$, apesar de ter havido tendência de aumento das CAPE 2 e 3 e também na tendência dos EEPRP, a correlação entre esses parâmetros foi fraca. Ou seja, a CAPE não pode ser usada como ferramenta de previsão climatológica para Belém-PA, pois CAPE é energia potencial disponível para formação da convecção úmida, que é inicialmente acumulada e liberada durante a PRP. Sendo assim, a energia gerada é consumida pela atividade das nuvens precipitantes, que transformam essa energia em correntes ascendentes.

A avaliação entre a frequência anual da CAPE com a variação de ION e GIHA mostrou não haver relação entre a CAPE e esses parâmetros, porque a correlação foi fraca, visto que, mesmo quando ocorre maior frequência da CAPE 1 para o ION e GIHA negativo (OAS quente), a correlação foi fraca, pois sempre quando chove a CAPE é consumida e a instabilidade é reduzida. Sendo assim, nos anos que foram caracterizados por grande quantidade de precipitação na cidade, a CAPE diminuiu. Confirmando novamente que a simples existência de CAPE na atmosfera não é condição suficiente para que ocorra a formação de nuvens e precipitação, já que a forçante dinâmica de grande escala modula o clima da região.

Ficou claro que é necessário fazer ajuste nos valores de referência para classificação da CAPE, para Belém-PA, já que nesta cidade a temperatura é elevada, o que impacta nos valores

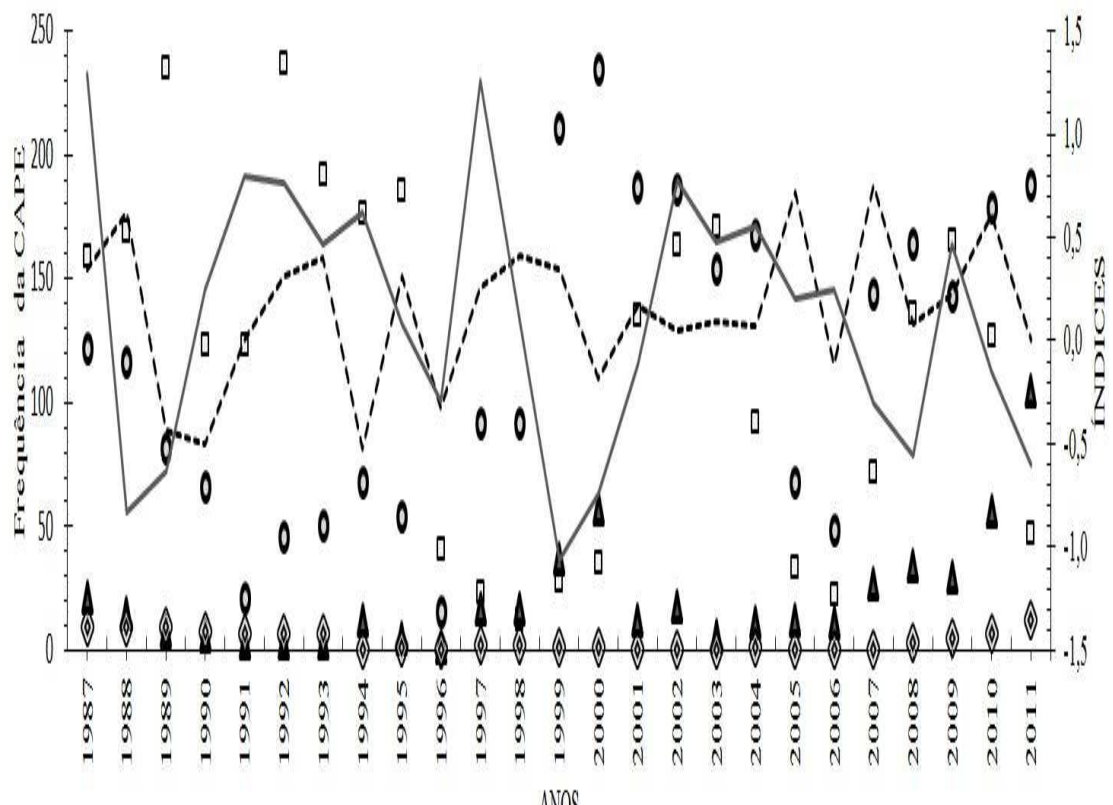

AOS

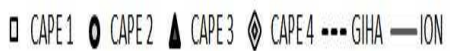

Figura 7 - Frequência anual das categorias da CAPE para Belém-PA e média anual dos índices oceânicos GIHA e ION no período de 1987 a 2011 


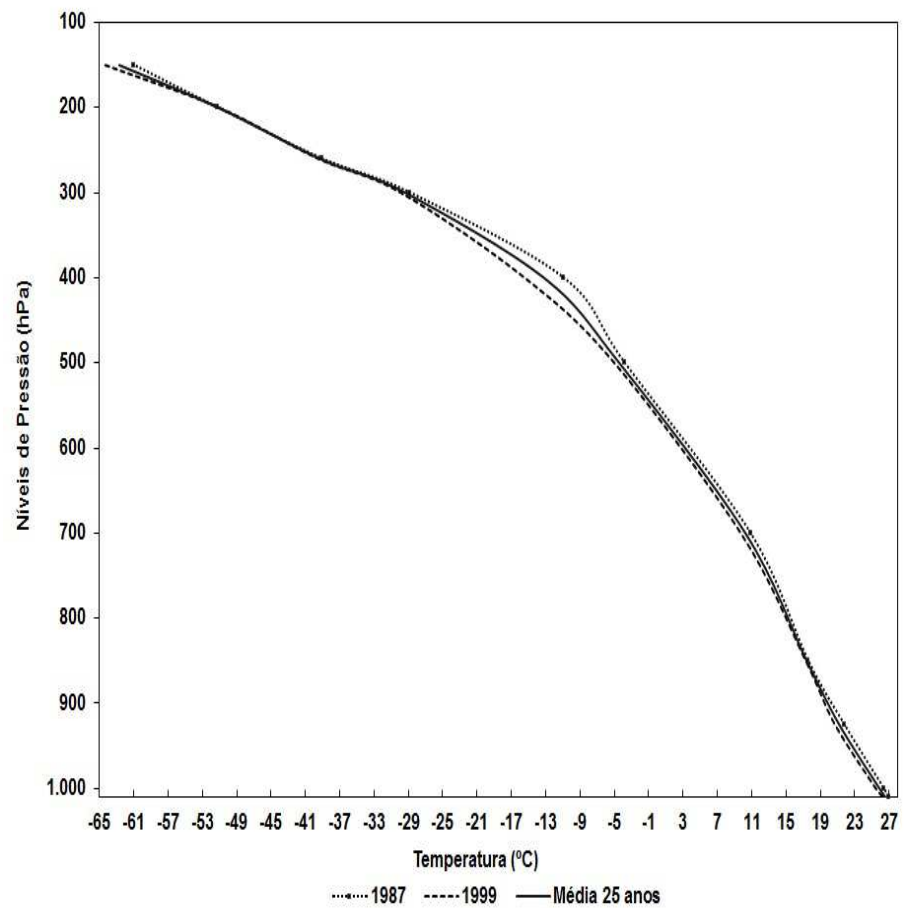

Figura 8 - Perfis verticais das temperaturas da atmosfera de Belém-PA, para os anos de 1987, 1999 e média dos 25 anos estudados.

da CAPE, pois nem sempre altos/baixos valores de CAPE coincidem com aumento/diminuição da PRP.

\section{AGRADECIMENTOS}

Os autores agradecem à Financiadora de Estudos e Projetos (FINEP) pelo financiamento do Projeto REMAM.

\section{REFERÊNCIAS BIBLIOGRÁFICAS}

ANANIAS, D. S. et al. Climatologia da estrutura vertical da atmosfera em novembro para Belém-Pa. Revista Brasileira de Meteorologia, v. 25, n. 2, p. 218 - 226, 2010.

CAVALCANTI, I. F. A.; FERREIRA, N. J.; SILVA DIAS, M. A. F.; JUSTI, M. G. A. Tempo e Clima no Brasil. Oficina de Textos, p. 25-27, p. 75-93. São Paulo, 2009.

CHARNEY, J. Dynamics of deserts and drought in the Sahel. Quarterly Journal of the Royal Meteorological Society, v. 101, p. 193-202. 1975.

CITEAU, J.; BERGES, J. C.; DEMARCQ, H.; MAHÉ, G. Position de la Zone de Convergence a $28^{\circ} \mathrm{N}$ et temperatue de surface de 1'ocean. Veille Climatique Satellitaire, v. 21, p. 3-7, 1988a.

CITEAU, J.; BERGÉS, J. C.; DEMARCQ, H.; MAHÉ, G. The watch of ITCZ migrations over tropical Atlantic as an indicator in drought forecast over Sahelian area. OceanAtmosphere Newsletter, v.45, p.1-3. 1988b.
CITEAU, J. et al. Questions about the ITCZ migration over the tropical Atlantic, sea surface temperature in the Gulf of Guinea and the Flow of Senegal River. In: CCCO Meeting, Rio de Janeiro, 1985.

COELHO, C. A. S.; DRUMOND, A. R. M.; AMBRIZZI, T. Estudo climatológico sazonal da precipitação sobre o Brasil em episódios extremos da Oscilação Sul. Revista Brasileira de Meteorologia, v. 14, n. 1, p. 49-65, 1999.

COHEN, J. C. Statistical power analysis for the behavioral sciences. Hillsdale, NJ, Erlbaum. 1988.

COHEN, J. C.; SILVA DIAS, M. A. F.; NOBRE, C. Aspectos climatológicos das linhas de instabilidade na Amazônia. Climanálise, v. 4, n. 11, p. 34-40. 1989.

COSTA, A. C. L. Estudo de Variações Termo-Higrométricas de Cidade Equatorial devido ao Processo de Urbanização. O caso de Belém - PA, 1998, 232f., Tese. Universidade de São Paulo, São Carlos, SP. 1998.

COX. P.; et al. Increase risk of Amazonian Drought due to decreasing aerosol pollution. Nature (London), v. 453, p. 212 - 216, 2008.

CRAVEN, J. P.; JEWELL, R. E.; BROOKS, H. E. Comparison between observed convective cloud-based heights and lifting condensation level for two different lifted parcels. Weather Forecasting, v. 17, p. 885-890, 2002.

DE SOUZA, E. B.; AMBRIZZI, T. ENSO impacts on the South American rainfall during 1980s: Hadley and Walker circulation. Atmósfera, v. 15, p. 105-120, 2002. 
DE SOUZA, E. B. et al. On the influences of the El Niño, La Niña and Atlantic dipole pattern on the Amazonian rainfall during 1960-1998. Acta Amazonica, v. 30, n. 2, p. $305-$ $318,2000$.

DONNER, L.; PHILLIPS, V. Boundary layer control on convective available potential energy: Implications for cumulus parameterization. Journal of the Geophysical Research, v. 108, (D22) doi:10.1029/2003JD003773, 2003.

DOSWELL, C.; RASMUSSEN, E. The effect of neglecting the virtual temperature correction on CAPE calculations. Weather and Forecasting, v. 9, p. 625-629, 1994.

EMANUEL, K. A.; NEELIN, J. D.; BRETHERTON, C. S. On large-scale circulations in convecting atmosphere. Quarterly Journal of the Royal Meteorological Society, v. 120, n. 519, p. 1111-1143, 1994.

FIGUEROA, S. N.; NOBRE, C. A. Precipitations distribution over Central and Western Tropical South America. Climanálise-Boletim de Monitoramento e Análise Climática, v. 5, n. 6, p. 36-48, 1990.

GETTELMAN, A.; SEIDE, D. J.; WHEELER, M. C.; ROSS, R. S. Multidecadal trends in tropical convective available potential energy. Journal of Geophysical Research, v. 107, p. 4606-4614, 2002.

GOLDEN GATE WEATHER SERVICES. ENSO Years based on Oceanic Niño Index (ONI). Disponível em: $<\mathrm{http}: / /$ ggweather.com/enso/oni.htm>. Acessoem: março de 2014.

HASTENRATH, S.; LAMB, P. Some aspects of circulation and climate over the eastern tropical Atlantic. Monthly Weather Review, v. 105, p. 1019-1023, 1977.

KAYANO, M. T.; MOURA, A. D. O El Nino de $1982-83$ e a precipitação sobre a América do Sul. Revista Brasileira de Geofísica, v. 4: p. 201-214, 1986.

KOUSKY, V. E.; CAVALCANTI, I. F. A.; KAYANO, M. T. A review of the Southern Oscillation: oceanic-atmospheric circulation changes and related rainfall anomalies. Tellus, v. 36A, p. 490-504, 1984.

LIEBMANN, B.; MARENGO, J. A. Interannual variability of the rainy season and rainfall in the Brazilian Amazon Basin. Journal of Climate, v. 14, p. 4308-4318, 2002.

LIMA, D. R. O. "Diagnóstico de chuvas e previsão meteorológica para a bacia hidrográfica do rio Manso". Tese-Universidade Federal do Rio de Janeiro, COPPE, RJ, Brasil. 2005.

MACHADO, L. A. T.; LAURENT, H.; LIMA, A. Diurnal march of the convection observed during TRMM-WETAMC/ LBA. Journal of the Geophysical Research, v.107, p. 8064, 2002, DOI: 10.1029/2001JD000338.

MARENGO, J. A. Interannual variability of surface climate in the Amazon basin. International Journal of Climatology, v. 12, p. 853-863, 1992.
MARENGO, J. A.; et al. The drought of Amazônia in 2005. Journal of Climate. v. 21, p. 495 - 516, 2008 a.

MARENGO, J. HASTENRATH, S. Case studies of extreme climatic events in the Amazon Basin. Journal of Climate, v. 6, n. 4, p. 617-627, 1993.

MARENGO J. A.; LIEBMANN, B.; KOUSKY, V.; FILIZOLA, N. S.; WAINER, I. . On the onset and end of the rainy season in Brazilian Amazon basin. Journal of Climate, Lancaster, PA, v. 14, p. 833-852, 2001.

MARENGO, J. A.; NOBRE, C. A.; TOMASELLA, J.; CARDOSO, M. F.; OYAMA, M. D. Hydro-climatic and ecological behavior of the drought of Amazônia in 2005. Philosophical Transactions of the Royal Society of London. Series A. Mathematical and Physical Sciences, v. 21B. pp 1-6. 2008b.

MCBRIDE, J.; FRANK, W. Relationships between stability and monsoon convection. Journal of the Atmospheric Sciences, v. 56, p. 24-36, 1999.

MOTA, M. A. S. Convecção na Amazônia: variabilidade, efeitos e respostas na circulação de Grande escala. Tese de doutorado em Meteorologia. Instituto Nacional de Pesquisas Espaciais. São José dos Campos - São Paulo. 2004.

MOTA, M. A. S.; NOBRE, C. A. Relação da variabilidade da energia potencial convectiva disponível (CAPE) com a precipitação e a alta da Bolívia durante a campanha WetAMC/LBA. Revista Brasileira de Meteorologia, v. 21, $\mathrm{n}$. 3b, p. 344-355, 2006.

MURUGAVEL, P., PAWAR, S. D.; GOPALAKRISHNAN, V. Trends of Convective Available Potential Energy over the Indian region and its effect on rainfall. International Journal of Climatology, v. 32: p. 1362-1372, 2012. doi: $10.1002 /$ joc. 2359 .

NASCIMENTO, E. L., "Previsão de tempestades severas utilizando-se parâmetros convectivos e modelos de meso escala: uma estratégia operacional adotável no Brasil? Revista Brasileira de Meteorologia, v. 20, n. 1, p. 121140, 2005.

NOBRE, P.; SHUKLA, J. Variations of sea surface temperature, wind stress, and rainfall over the tropical Atlantic and South American. Journal of Climate, v. 9, p. 2464-2479, 1996.

PETERSEN, W. et al. Intraseasonal Forcing of Convection and Lightning Activity in the Southern Amazon as a Function of Cross-Equatorial Flow. Journal of Climate, v. 19, p.31803196, 2006.

PETERSEN, W. et al. TRMM Observations of Intraseasonal Variability in Convective Regimes over the Amazon. Journal of Climate, v. 15, p.1278-1294, 2002.

PEZZI, L. P.; CAVALCANTI, I. F. A. The relative importance of ENSO and tropical Atlantic sea surface temperature anomalies for seasonal precipitation over South America: 
a numerical study. Climate Dynamics, v. 17, p. 205-212, 2001.

RENNÓ, N. O.; INGERSOLL, A. P. Natural Convection as a Heat Engine: A theory for CAPE. Journal of the Atmospheric Sciences. v.. 53, p. 571 - 585, 1996.

ROCKWOOD, A. A.; MADDOX, R. A. Mesoscale and synoptic scale interactions leading to intense convection: The case of 7 June 1982. Weather and Forecasting, v. 3, p. 51-68, 1988.

ROGASH, J. A.; RACY, J. Some Meteorological Characteristics of Significant Tornado Events occurring in proximity to Flash Flooding. Weather and Forecasting. v. 17, n. 2, p.155-200, 2002.

RUTLEDGE, S. A.; PETERSEN, W. A.; CIFELli, R. C.; CAREY, L.D. Early results from TRMM-LBA: Kinematic and microphysical characteristics of convection in distinct meteorological regimes. In: AMS $24^{\text {th }}$ Conf. On Hurricanes and Tropical Meteorology, Ft. Lauderdale, FL, 2000.

SALATI, E.; MARQUES, J. Climatology of the Amazon region. In The Amazon - Limnology and landscape ecology of a mighty tropical river and its basin. Sioli, H. (ed.). Dr. W. Junk Publishers, pp.763, 1984.

SILVA DIAS, M. A. F. "Índices de Instabilidade para Previsão de Chuva e Tempestades Severas", Universidade de São Paulo, SP, 2000.

SILVA JÚNIOR, J. A.; COSTA, A. C. L.; PEZZUTI, J. C. B.; COSTA, R. F.; GALBRAITH, D. Análise da Distribuição Espacial do Conforto Térmico na Cidade de Belém, PA no Período Menos Chuvoso. Revista Brasileira de Geografia Física, v.2, p. 218-232, 2012.

SHIMSHONI, M. On Fisher's test of significance in harmonic analysis. Geophys. Journal of the Royal Astronomical Society of Canada, v. 23, p. 373-377, 1971.

SNEYERS, R. On the use of Statistical Analysis for the Objective Determination of Climatic Change. Meteorologische Zeitschrift, v.1, p. 247-256, 1992.

SOUSA, E. P. P. Relações entre as anomalias de TSM do Atlântico e Pacífico e as precipitações na Amazônia oriental. Dissertação de Mestrado do Curso em Meteorologia. INPE, 2003.

STULL, R. B. An introduction to boundary layer meteorology. Dordrecht: Kluwer, 1988.

TAVARES, J. P. N.; MOTA, M. A. S. Condições termodinâmicas de eventos de precipitação extrema em Belém-Pa durante a estação chuvosa. Revista Brasileira de Meteorologia, v. 27, n.2, p. 207 - 218, 2012.

UVO, C. B.; NOBRE, C. A. A Zona de Convergência Intertropical (ZCIT) e a precipitação no norte do Nordeste do Brasil. Parte I: A posição da ZCIT no Atlântico equatorial.
Climanálise, São José dos Campos, v. 4, n. 7, p. 34-42, 1989.

WALISER, E. W. C.; GAUTIER, C. A Satellite-derived Climatology of the ITCZ. Journal of Climate, v. 6. p. 2162-2174, 1993.

WILLIAMS, E., et al. A radar and electrical study of tropical "hot towers." Journal of the Atmospheric Sciences. v. 49, p. 1386-1395, 1992.

WILLIAMS, E., et al. Contrasting convective regimes over the Amazon: Implications for cloud electrification. Journal of the Geophysical Research, v. 107, 2002, doi:10.1029/2001JD000380.

WILLIAMS, E.; RENNÓ, N. O. An Analysis of the conditional instability of the Tropical atmosphere. Monthly Weather Review, v. 121, n.1, p.21-36. 1993.

XAVIER, T. M. B. S. el al. Aspectos Climáticos de Belém nos Últimos Cem Anos. Belém: Embrapa Amazônia Oriental, 2002. Disponível em: $<\mathrm{http}: / / w w w . i n f o t e c a . c n p t i a . e m b r a p a$. br/bitstream/doc/389773/1/OrientalDoc128.pdf $>$. Acesso em julho de 2013.

XAVIER, T. M. B. S; XAVIER, A. F. S. Caracterização de períodos secos ou excessivamente chuvosos no estado do Ceará através da técnica dos quantis : 1964-1998. Revista Brasileira de Meteorologia, v.14, n.2,63-78,1999.

XAVIER, T.M.B.S.; XAVIER, A. F. S.; ALVES, J. M. B. Quantis e eventos extremos - aplicações em ciências da terra e ambientais. Fortaleza: RDS. Editora, Livrarias Livros Técnicos, 278p. 2007.

YANO, J.; CHABOUREAU, J.; GUICHARD, F. A generalization of CAPE into potential energy convertibility. Quarterly Journal of the Royal Meteorological Society, v. 131, p. 861-875, 2005.

YE, B.; DEL GENIO, A. D.; LO, K. K. W. CAPE variations in the current climate and in a climate change. Journal of Climate, v. 11, n. 8, p.1997-2015, 1998.

ZENG N., YOON, J. H, MARENGO, J. A.; SUBRAMANIAM A.; NOBRE, C. A.; MARIOTTI A et al. Causes and impacts of the 2005 Amazon drought. Environmental Research Letters, v. 3, n. 1, p. 1-9, 2008.

ZHANG, G. Convective quasi-equilibrium in the tropical western Pacific: comparison with mid latitude. Journal of the Geophysical Research, v. 108, 2003a, doi:10.1029/ 2003JD003520.

ZHANG, G. Roles of tropospheric and boundary layer forcing in the diurnal cycle of convection in the U.S. southern Great Plains. Geophysical Research Letters, v. 30, 2003b, doi:10.1029/ 2003JD018554. 